\title{
Seguridad y salud en el trabajo en Ecuador
}

\section{Safety and Health at Work in Ecuador}

Antonio Ramón Gómez Garcíaํㅜㅇ 0000-0003-1015-1753

${ }^{1}$ Facultad de Postgrado, Universidad de Especialidades Espíritu Santo, Samborondón, Ecuador.

Fechas · Dates

Recibido: 2021.04.11

Aceptado: 2021.04.11

Publicado: 2021.07.15
Correspondencia · Corresponding Author

Antonio Ramón Gómez García

Correo electrónico: agomezg@uees.edu.ec 
La República del Ecuador (en adelante Ecuador) puede ser considerada como uno de los países latinoamericanos más privilegiados por sus contrastes geográficos, variedad climática y amplia biodiversidad. La Cordillera de los Andes divide al Ecuador en tres regiones (Amazonía, Sierra y Costa del Pacífico) con marcadas diferencias culturales. Además, existen el conjunto de las Islas Galápagos como cuarta región. La población (2020) es de 17,5 millones de habitantes (51\% son mujeres) y la esperanza de vida aumentará significativamente en los próximos años (2010 fue de 75 años y para el 2030 será de 80,5 años) $)^{(1)}$.

En contraste, los indicadores de salud, económicos y laborales muestran un paronarma preocupante. La tasa de mortalidad en los últimos años ha aumentado ligeramente, en 2014 fue de 4,1 por cada 1000 habitantes y en 2019 fue de 4,3 por cada 1000 habitantes. En 2019, la primera causa de muerte fueron las enfermedades isquémicas del corazón, tanto en mujeres y hombres, y en adultos de 30 a 64 años los accidentes de transporte ${ }^{(2)}$. Aunque la economía de Ecuador ha mostrado un crecimiento hasta mediados de la última década, la caída del precio internacional del petróleo provocó una recesión económica, con pérdidas de plazas de empleo adecuado desde 2015 y que se mantienen progresivamente en la actualidad. El empleo informal ha crecido rápidamente, situándose en el 46,7\% en 2019, y en condiciones precarias de trabajo y ausencia derechos de protección social(3). Desafortunadamente, la crisis sanitaria por el SARS-CoV-2 ha provocado aún más un entorno desfavorable y retroceso en los objetivos marcados para reducir las desigualdades sociales.

En el período comprendido entre 2006 y 2020, y desde que se disponen datos en el Seguro General de Riesgos de Trabajo (SGRT) del Instituto Ecuatoriano de Seguridad Social (IESS), han sucedido hitos diferenciados en la evolución y tendencias de la siniestralidad laboral en el país. El primer precedente se encuentra en la Resolución 741 de 1990(4), creada para establecer los criterios de concesión de prestaciones por accidentes de trabajo y enfermedades profesionales en los trabajadores afiliados. El segundo gran hito fue la aparición de dos resoluciones consecutivas, la Resolución 333 de 2010(5) y la Resolución 390 de 2017(6). En ambas resoluciones, se estableció la necesidad de implementar sistemas de gestión de seguridad y salud en el trabajo en las empresas que permitiera reducir o eliminar los riesgos laborales presentes en los lugares de trabajo, asimismo, la obligatoriedad de reportar los accidentes de trabajo y posibles enfermedades profesionales al SGRT. Finalmente, las dos resoluciones anteriores fueron sustituidas en marzo de 2016 por la vigente Resolución $513^{(7)}$, si bien, se mantienen las premisas de prevención y aparece la necesidad de promoción de la salud, se eliminaron por completo las auditorías de los sistema de gestión y se modificaron, sin mayores cambios, las atribuciones de responsabilidad patronal.

En consecuencia, es probable que los cambios constantes en los órdenes jurídicos pudieran haber influenciado significativamente en los datos mostrados en las Figuras 1 y 2. Con una perspectiva de 15 años, las tasas de incidencia de accidentes de trabajo (mortales y no mortales) y enfermedades profesionales (por cada 1.000 trabajadores afiliados) muestran vaivenes con tendencias al aumento 
(2010-2015) y a la dimisminucion (2016 y 2017-2019), siendo el 2020 un año atípico debido a la pandemia por la COVID-19. Además, las cifras expuestas podrían estar influenciadas por dos factores importantes. Por un lado, la población trababajadora con cobertura del seguro por riesgos del trabajo ha diminuido desde $2015^{(9)}$ y, por otro, el problema del sub-registro de accidentes de trabajo y enfermedades profesionales puedan enmascarar la realidad y, por tanto, no sería exacto afirmar una mejora de las condiciones de trabajo reflejados en los indicadores de siniestralidad laboral del país.
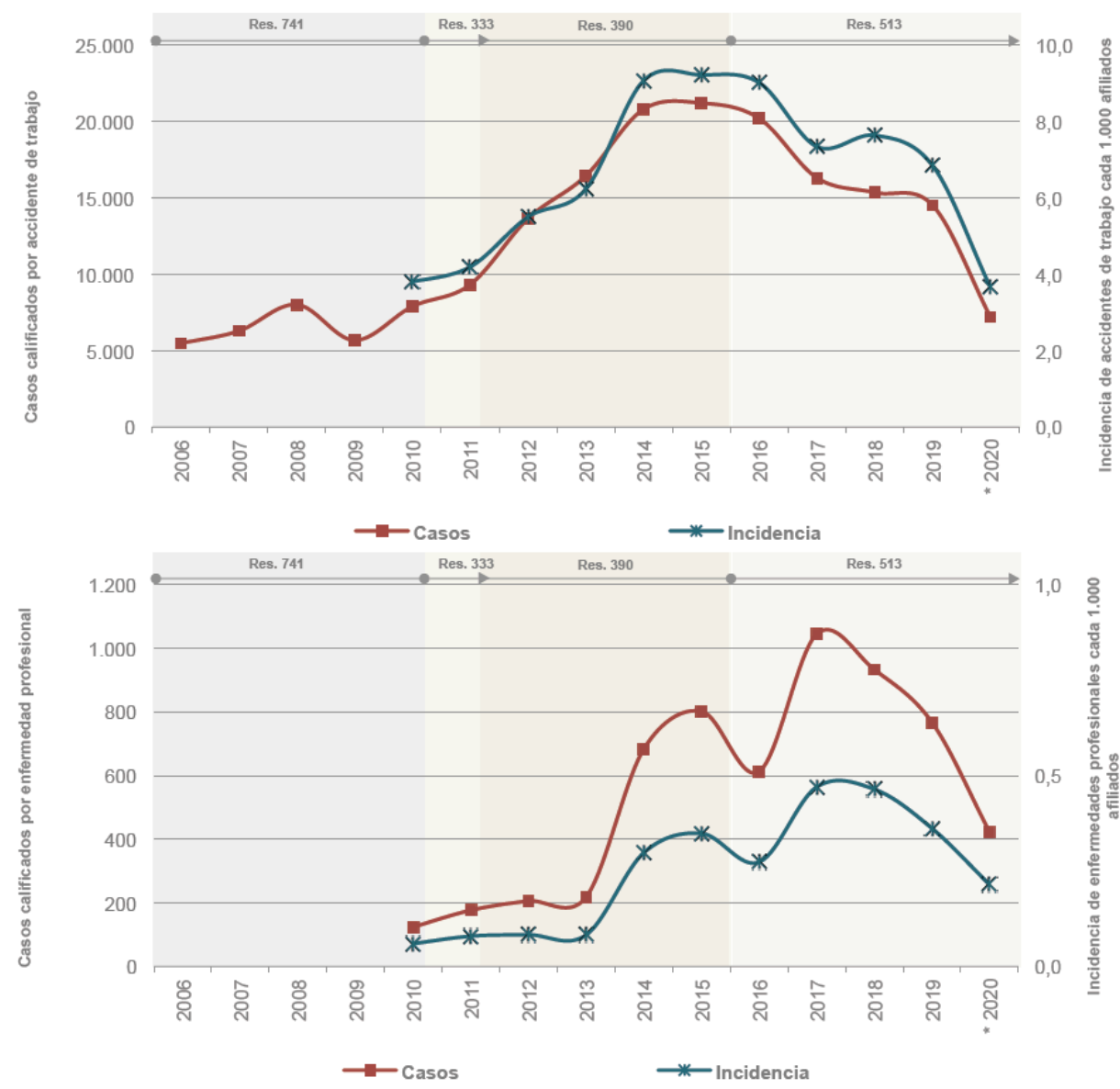

Figura 1: Evolución de los casos y la incidencia de accidentes de trabajo mortales y no mortales calificados en la República del Ecuador. Periodo 2006-2020 (24 de diciembre de 2020).

Fuente: elaboración propia, a partir de datos facilitados por el Seguro General de Riesgos de Trabajo y anuario ${ }^{(8)}$ y población trababajadora con cobertura del seguro por riesgos del trabajo ${ }^{(9)}$. 

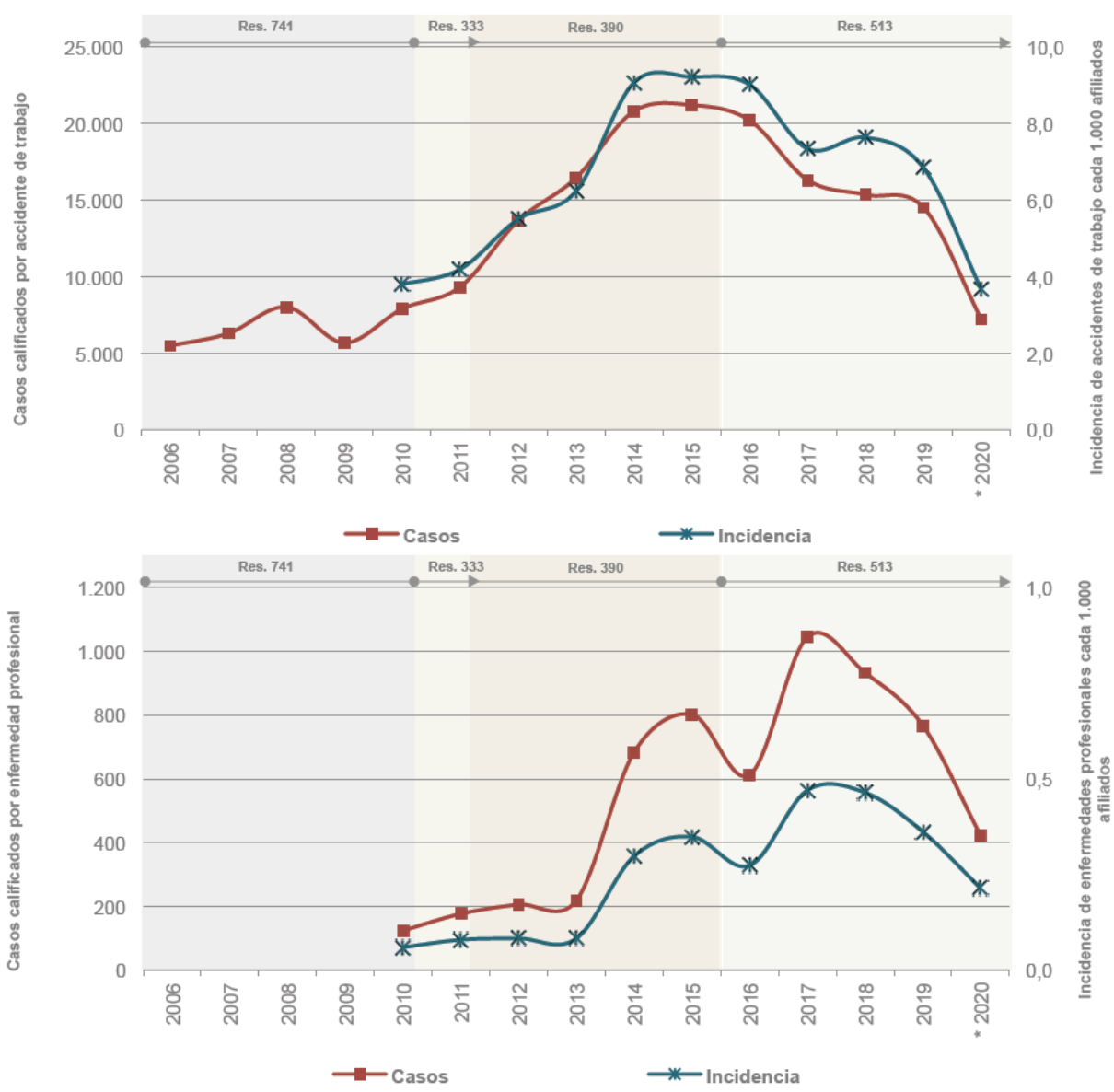

Figura 2: Evolución de los casos y la incidencia de enfermedades profesionales calificados en la República del Ecuador. Periodo 2010-2020 (24 de diciembre de 2020).

Fuente: elaboración propia, a partir de datos facilitados por el Seguro General de Riesgos de Trabajo y anuario( ${ }^{(8)}$ y población trababajadora con cobertura del seguro por riesgos del trabajo(9).

Un aspecto a reseñar, que también podría incidir en este contexto, es el número reducido de inspectores de trabajo a nivel nacional (12 técnicos y 1 médico del trabajo habilitados) que componen la Dirección de Seguridad, Salud en el Trabajo y Gestión Integral de Riesgos del Ministerio del Trabajo. En el país se estima 0,04 inspectores por cada 10 mil trabajadores, cifra inferior al promedio de América Latina y el Caribe que varía signifcativamente desde 0,1 a 1,9 inspectores por cada 10 mil trabajadores ${ }^{(11)}$. En este sentido, se dificulta la comprobación del cumplimiento y control de la aplicación de la normativa de prevención de riesgos laborales en la empresas, principalmente, en aquellas provincias de la región amazónica más desfavorecidas y con menor presencia de inspectores. A ello habría que añadir la necesidad de crear un Cuerpo de Inspectores con nombramiento (funcionarios públicos) y la adecuada formación continua para garantizar un sistema eficaz de inspección como elemento central que garantice el trabajo decente ${ }^{(11,12)}$. Por tanto, 
el problema entre la normativa existente y su cumplimiento continuará siendo un desafío para el país.

La oferta formativa de las universidades en pregrado y postgrado relacionada con la seguridad y salud en el trabajo (SST) ha aumento significativamente en la última década, aspecto similir a lo observado en países cercanos a Ecuador ${ }^{(13,14)}$. Al momento actual, existen vigentes 12 programas de maestrías y 3 carreras universitarias en las instituciones de educación superior. No obstante, en diciembre de 2020 tuvo lugar el Seminario Internacional sobre la Calidad de los Posgrados organizado por Consejo de Educación Superior (órgano regulador de la educación superior) y se pudo constatar heterogeneidades en las características académicas y los meso-micro currículos entre los programas de maestrías relacionados con la SST, así como, cierta variabilidad en las denominaciones de los títulos académicos y asignaturas, carga lectiva en horas totales y perfiles de egreso ${ }^{(15)}$. Además, se desconoce el numero de profesionales técnicos, especialistas en medicina y enfermería del trabajo que ofrezca información precisa para conocer la demanda real de estos profesionales y especialistas en el mercado laboral, aunque seguramente sean muy necearios. En este sentido, se añade la falta de reconocimiento social de los profesionales en SST, a pesar convertirse en una prioridad en salud pública y laboral desde el comienzo de la pandemia por COVID-19.

Las investigación, hasta ahora, resulta ser poco significativa para permitir un conocimiento general de las condiciones de trabajo y su afectación a la salud de la población trabajadora ecuatoriana ${ }^{(16-18)}$. En este sentido, podría estar explicado por la inexistente cultura investigativa, incluso después de las incitativas de fomento a la investigación por el gobierno ecuatoriano en los útlimos años. Dado que las encuestas sobre condiciones de trabajo, empleo y salud (ECTES) suponen una herramienta de gran utilidad para obtener información fiable para generar conocimiento sobre la compleja relación entre el trabajo y la salud(19), la Universidad Internacional SEK (UISEK) y la Universidad de Especialidades Espíritu Santo (UEES) aplicaron la Primera Encuestas sobre Condiciones de Seguridad y Salud en el Trabajo (I-ECSST) en las dos principales ciudades del país (Quito y Guayaquil) en 2016 y 2017, respectivamente.

Los resultados de la I-ECSST, además de ser empleados como referentes para el diseño de la Política Nacional de Salud en el Trabajo 2019-2025(20), destacan la exposición a movimientos repetitivos (60\%) y la alta prevalencia de molestias de espalda (50\%), cabeza (40\%) y miembros superiores (26\%) en los trabajadores encuestados. Sin embargo, este conocimiento no representan la realidad nacional y el conjunto de ítems contenidos en la I-ECSST limita comparaciones más amplias con otros países de la Región. En este sentido, se evidencia la necesidad de conciliar y mejorar las ECTES en América Latina(21). Un futuro prometedor, consistiría en la participación activa del Observatorio Iberoamericano de Seguridad y Salud en el Trabajo (OISST), de reciente creación por la Organización Iberoamericana de Seguridad Social (OISS), servirá como punto de encuentro y colaboración del diagnóstico de la SST en los países de la Región. 
Y finalmente, mientras todo esto sucede, diferentes gremios conocidos por su antigüedad como la Sociedad Médica Ecuatoriana de Salud Ocupacional (SOMESO) y la Sociedad Ecuatoriana de Seguridad y Salud Ocupacional (SESO) han aportado iniciativas significativas en los últimos años. Igualmente destacan, por orden de importancia, la Asociación Ecuatoriana de Seguridad y Salud Ocupacional (AESSO), la Asociación de Profesionales en Prevención de Riesgos Laborales y Sistemas Integrados de Gestión (APPSIG) y la Asociación de Profesionales de Seguridad y Salud en el Trabajo en Ecuador (APSSTEC), aunque estas asociaciones trabajan por caminos separados y de forma intermiente. Las opiniones desde la experiencia son de gran valor para la propuesta de acciones públicas en SST ${ }^{(20)}$, a pesar de no ser considerados argumentos sólidos o contrastados.

En resumen, y teniendo en cuenta todo lo expuesto anteriormente, impera la necesidad de estudiar en profundidad y de manera sistemática las condiciones de trabajo, empleo y salud, tanto de los que trabajan en el sector formal como informal, incorporando indicadores socioeconómicos y de salud y en colaboración con redes multidisciplinaras e internacionales. En definitiva, además de crear conciencia y sensibilización, Ecuador asume una ardua tarea en generar conocimiento que permita impulsar políticas públicas para los próximos años y favorecer la mejora de la calidad de vida de la población trabajadora ecuatoriana.

\section{Bibliografía}

1. Instituto Nacional de Estadística y Censos (INEC). Proyecciones de la Población de la República del Ecuador 2010-2050. Quito; 2012. [citado: 3 marzo 2021]. Disponible en: https://www.ecuadorencifras.gob.ec/proyecciones-poblacionales.

2. Instituto Nacional de Estadística y Censos (INEC). Registro Estadístico Defunciones Generales 1990-2019. Quito; 2020. [citado: 4 marzo 2021]. Disponible en: https://www.ecuadorencifras.gob.ec/documentos/web-inec/Poblacion_y_Demografia/Defunciones_Generales_2019/Presentacion_EDG\%20_2019.pdf.

3. Comisión Económica para América Latina y el Caribe (CEPAL). Bases de Datos y Publicaciones Estadísticas CEPALSTAT, Ecuador: perfil nacional económico. Santiago de Chile; 2021. [citado: 7 marzo 2021]. Disponible en: https://estadisticas.cepal.org/cepalstat/Perfil_Nacional_Economico.html?pais=ECU\&idioma=spanish.

4. Instituto Ecuatoriano de Seguridad Social (IESS). Resolución C.D. No. 741; Reglamento General del Seguro de Riesgos del Trabajo. Quito; 1990.

5. Instituto Ecuatoriano de Seguridad Social (IESS). Resolución C.D. No. 333; Reglamento para el Sistema de Auditoría de Riesgos del Trabajo - SART. Quito; 2010.

6. Instituto Ecuatoriano de Seguridad Social (IESS). Resolución C.D. No. 390. Reglamento del Seguro General de Riesgos del Trabajo. Quito; 2011.

7. Instituto Ecuatoriano de Seguridad Social (IESS). Resolución C.D. No. 513; Reglamento del Seguro General de Riesgos del Trabajo. Quito; 2016. 
8. Gómez García AR, Suasnavas Bermúdez PR, Freire Constante LF, Trujillo Otáñez DA. (2016). Accidentes de Trabajo y Enfermedades Profesionales en Ecuador: 2006-2014. Cuenca: Editorial Don Bosco.

9. Instituto Nacional de Estadística y Censos (INEC). Encuesta Nacional Empleo, Desempleo y Subempleo - ENEMDU. Quito; 2021. [citado: 5 marzo 2021]. Disponible en: https://www.ecuadorencifras.gob.ec/empleo-enero-2021.

10. Ronconi L. Globalization, domestic institutions and enforcement of labor law: evidence from Latin America. Journal of Economy and Society. 2012;51:89-105. doi:10.1111/j.1468-232X.2011.00664.x

11. Bensusán G. La Inspección del Trabajo en América Latina: teorías, contextos y evidencias. Estudios Sociológicos. 2009;27(81):989-1040.

12. Organización Internacional del Trabajo (OIT). La economía informal y el trabajo decente: una guía de recursos sobre políticas, apoyando la transición hacia la formalidad. Ginebra: 2013. [citado: 7 marzo 2021]. Disponible en: https://www. ilo.org/wcmsp5/groups/public/---ed_emp/---emp_policy/documents/publication/ wcms_212689.pdf.

13. Cruz I, Huerta-Mercado R. Occupational Safety and Health in Peru. Ann Glob Health. 2015;81(4):568-75. doi: 10.1016/j.aogh.2015.08.027.

14. Caraballo-Arias Y. Occupational Safety and Health in Venezuela. Ann Glob Health. 2015;81(4):512-21. doi: 10.1016/j.aogh.2015.08.022.

15. Gómez García AR, Salazar Uquillas PJ. Competencias y contenidos de los programas de maestrías en seguridad y salud en el trabajo en Ecuador. Nuevos retos y oportunidades. En: Seminario Internacional sobre la Calidad de los Posgrados. Quito: Consejo de Aseguramiento de la Calidad de la Educación Superior - CACES; 2020.

16. Gómez García AR, Merino-Salazar P, Silva-Peñaherrera M, Suasnavas Bermúdez PR, Vilaret Serpa A. I Encuesta sobre Condiciones de Seguridad y Salud en el Trabajo para Ecuador. Principales resultados en la ciudad de Quito, 2016. Medicina y Seguridad del Trabajo. 2019;65(257):238-251.

17. Silva-Peñaherrera M, Merino-Salazar $P$, Benavides FG., López-Ruiz M, Gómez García AR. La salud ocupacional en Ecuador: una comparación con las encuestas sobre condiciones de trabajo en América Latina. Rev. Bras. Saúde Ocup. 2020;45:e20. doi: 10.1590/2317-6369000010019

18. Gómez García AR, Portalanza Chavarría CA, Arias Ulloa CA, Espinoza Samaniego, César E. Salaried workers' self-perceived health and psychosocial risk in Guayaquil, Ecuador. Int. J. Environ. Res. Public Health. 2020;17(23):9099. doi:10.3390/ ijerph1723909

19. Merino-Salazar P, Artazcoz L, Campos-Serna J, Gimeno D, Benavides FG. National working conditions surveys in Latin America: comparison of methodological characteristics. Int J Occup Environ Health. 2015;21(3):266-74. doi: 10.1179/2049396715Y.0000000004 
20. Ministerio de Salud Pública (MSP). Política Nacional de Salud en el Trabajo 2019 -2025. Quito; 2019. [citado: 10 marzo 2021]. Disponible en: https://www.salud.gob.ec/wp-content/uploads/2019/10/MANUAL-DE-POLITICAS-final.pdf.

21. Benavides FG, Merino-Salazar P, Cornelio $C$, Assunção AA, Agudelo-Suárez AA, Amable $\mathrm{M}$, et al. Cuestionario básico y criterios metodológicos para las Encuestas sobre Condiciones de Trabajo, Empleo y Salud en América Latina y el Caribe. Cad Saude Publica. 2016;32(9):1-13. doi:10.1590/0102-311×00210715 